\title{
Direct Observation of Vacancies, Impurities, Adsorbed Heavy Metals, Cation Ordering, and Interface Structures in Minerals Using Aberration-Corrected STEM
}

\author{
Huifang Xu${ }^{1}$ (hfxu@geology.wisc.edu)
}

1. Department of Geoscience, University of Wisconsin-Madison, 1215 W. Dayton St., Madison, Wisconsin 53706, United States

Understanding crystal structures of nano-phases, vacancies and impurities in minerals are important to elucidate formation mechanism and subsolidus reactions of minerals and their host rocks. Aberrationcorrected Z-contrast imaging can provide chemical images with sub- $\AA$ resolution. Scanning transmission electron microscopy (STEM) uses a high-angle annular dark-field (HAADF) detector to give the most highly localized 1s Bloch state imaging. Z-contrast images are HAADF images with atomic resolution. Multiple diffraction effects that appear in high-resolution transmission electron microscopic (HRTEM) imaging can be eliminated or minimized in Z-contrast imaging, because Z-contrast imaging uses noncoherent elastically scattered electrons at high scattering angle. Using a HAADF detector and annular bright-field (ABF) detector, both a Z-contrast image and an annular bright-field image can be obtained simultaneously. Bright spots in the Z-contrast image correspond to positions of atom columns. The contrast is roughly reversed in ABF image. The intensity of Z-contrast images is dependent on the atomic number of atom and occupancy in the site through $\mathrm{Z}^{\mathrm{n}}$. The power $\mathrm{n}(\sim 2)$ depends on experimental conditions. Local composition and occupancy may be obtained from measured intensities.

We can obtain positions of atoms directly over a large range of thickness, with Z-contrast to help distinguish columns of different atoms and their occupancies along the beam direction. Crystal structures of nano-minerals and nano-precipitates can be solved by combining the Z-contrast images and $a b$-initio calculation using density functional theory (DFT) method [1-4]. Vacancies, impurities, adsorbed heavy atoms can be also revealed directly. Examples of vacancy ordering in Fe-bearing olivine (Fig. 1) and Fe-sulfides (Fig. 2), positions of Au atoms in Au-bearing arsenopyrite, crystal structure of G. P. zones within OPX (Fig. 2), stacking faults in anisotropic sphalerite, adsorbed heavy metals (e.g., $\mathrm{As}, \mathrm{Au}, \mathrm{U}$ ) on nano-minerals, intercalation of graphene layers within clays, and cation orderings in pyroxene (Fig. 2) and incommensurately modulated plagioclase feldspars will be presented. New results show that $\mathrm{Ca}-\mathrm{Na}$ ordering and structural polarity of subcells in an intermediate plagioclase with modulated structure. Neighboring lamellar domains with I1 symmetry are related by inversion twin operation, instead of anti-phase domain boundaries (or, APBs) as in all previously reported structure models $[5,6]$.

\section{References:}

[1] Lee, S., Shen, Z., and Xu, H. (2016) American Mineral. 101, 1986-1995.

[2] Shen, Konishi, H., Szlufarska, I., P. E. Brown, and Xu, H. (2014) American Mineral. 99, 1413-1419.

[3] Xu, H., Shen, Z., Konishi, H., Fu, P., and Szlufarska, I. (2014) American Mineral. 99, 881-889.

[4] Xu, H., (2015) Mineralogical Magazine, 79, 377-385.

[4] Xu, H. (2015) American Mineral. 100, 510-515.

[6] Xu, H., Jin, S., and Noll, B. C. (2016) Acta Crystallographica, B72, 904-915.

[7] The authors acknowledge fundings from the NSF, US DOE, and NASA Astrobiology Institute. 

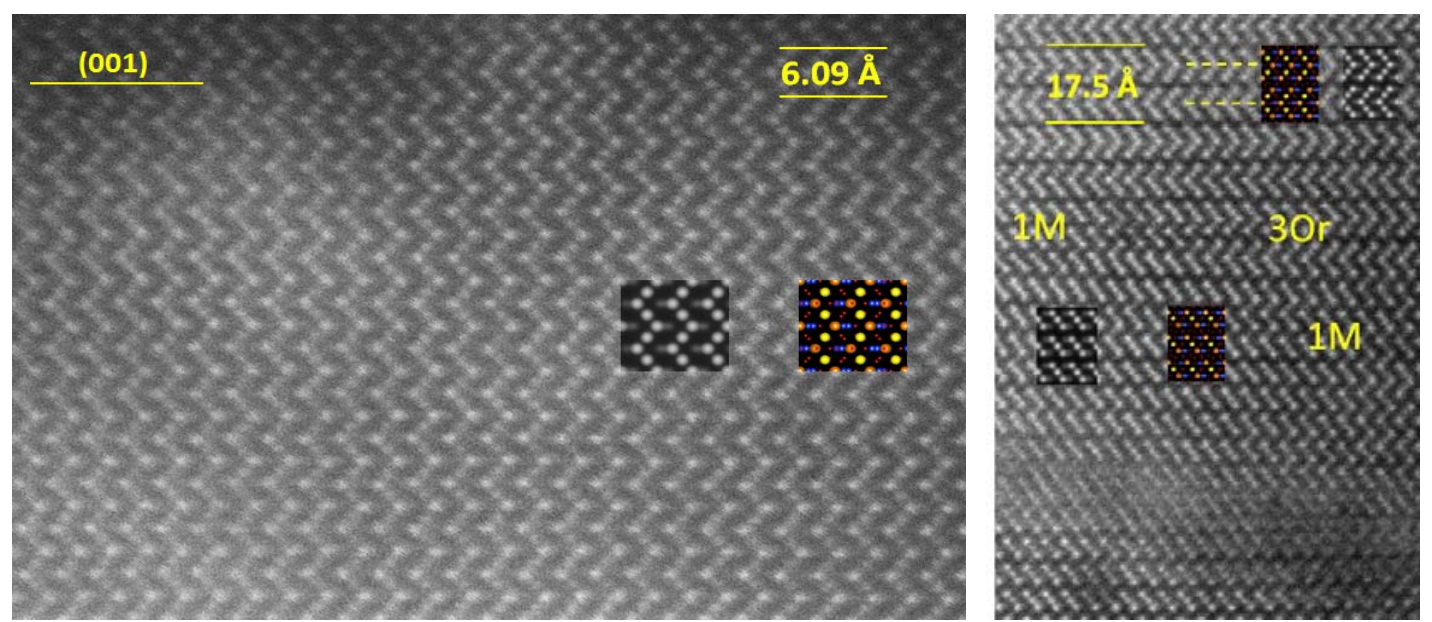

Figure 1. Left: Z-contrast image of fayalite ([110]-zone axis) shows positions of Fe in M1 (Fe+Mg) and M2 (Fe) sites. Fayalite model and simulated image are also overlaid on the image. The Fe atoms at M1, M2 sites, Si are colored in yellow, orange, and blue, respectively. Right: A [110]-zone-axis Z-contrast image showing intergrowth of 1-layer laihunite, []$_{0.5} \mathrm{Fe}_{0.5}^{2+} \mathrm{Fe}^{3+} \mathrm{SiO}_{4}\left(1 \mathrm{M}, P 2_{1} / b\right)$ and a 3-layer phase (3Or, Pbnm) with stoichiometry of []$_{1.0} \mathrm{Fe}_{3.0}^{2+} \mathrm{Fe}_{2.0}^{3+}\left[\mathrm{SiO}_{4}\right]_{3}$. Structure models and simulated images are also overlaid on the image. The 3-layer phase has same stoichiometry of a synthetic garnet "skiagite.”
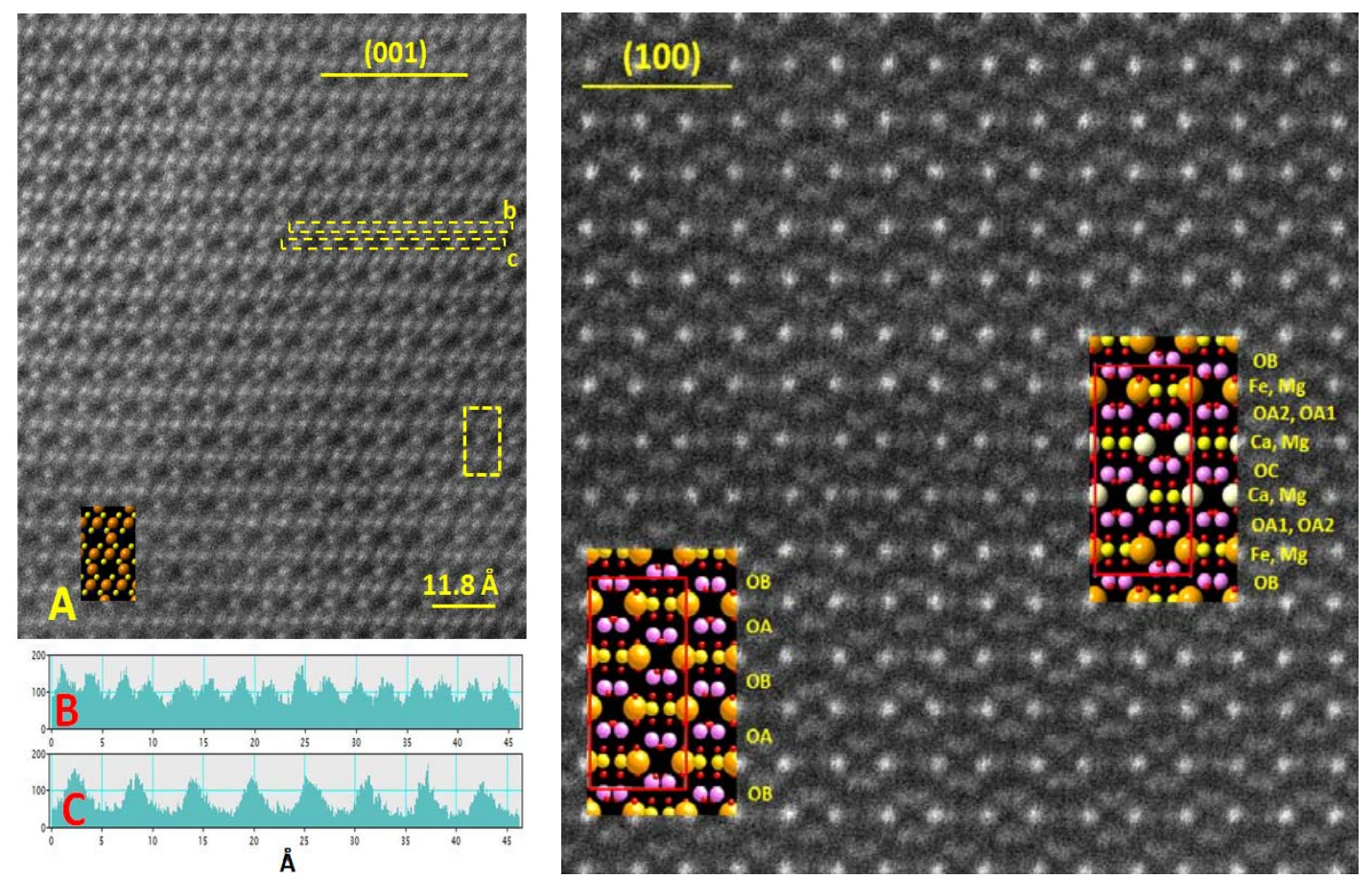

Figure 2. Left: High-magnification Z-contrast image of a monoclinic $\mathrm{Fe}_{3} \mathrm{~S}_{4}$, a polymorph of greigite along $\boldsymbol{b}$-axis showing positions of Fe columns, S columns, and vacancy columns. Projections of atoms based on the proposed structure model are also overlaid on the images. A high intensity peak in plot B indicate possible atom like $\mathrm{Ni}$ that is heavier than Fe. Right: Z-contrast image containing a G. P. zone with projections of the OPX structure and G. P. zone structure along [001] zone-axis. OA and OB tetrahedral chains are can be identified in the OPX host based on their appearance as "V" or dumbbell shapes. 